\title{
Thermospheric density variations: Observability using precision satellite orbits and effects on orbit propagation
}

\author{
Travis Lechtenberg, ${ }^{1}$ Craig A. McLaughlin, ${ }^{1}$ Travis Locke, ${ }^{1}$ and Dhaval Mysore Krishna ${ }^{1}$
}

Received 15 August 2012; revised 29 October 2012; accepted 27 November 2012; published 28 January 2013.

[1] This paper examines atmospheric density estimated using precision orbit ephemerides (POE) from the CHAMP and GRACE satellites during short periods of greater atmospheric density variability. The results of the calibration of CHAMP densities derived using POEs with those derived using accelerometers are examined for three different types of density perturbations, [traveling atmospheric disturbances (TADs), geomagnetic cusp phenomena, and midnight density maxima] in order to determine the temporal resolution of POE solutions. In addition, the densities are compared to High-Accuracy Satellite Drag Model (HASDM) densities to compare temporal resolution for both types of corrections. The resolution for these models of thermospheric density was found to be inadequate to sufficiently characterize the short-term density variations examined here. Also examined in this paper is the effect of differing density estimation schemes by propagating an initial orbit state forward in time and examining induced errors. The propagated POE-derived densities incurred errors of a smaller magnitude than the empirical models and errors on the same scale or better than those incurred using the HASDM model.

Citation: Lechtenberg, T. F., C. A. McLaughlin, T. Locke, and D. Mysore Krishna (2013), Thermospheric density variations: Observability using precision satellite orbits and effects on orbit propagation, Space Weather, 11, 34-45, doi:10.1029/2012SW000848.

\section{Introduction}

[2] The primary source of uncertainty for the orbit determination of low Earth-orbiting (LEO) satellites is atmospheric drag caused by the impact of atmospheric particles upon the spacecraft surface. In February 2009, two satellites collided above Siberia at a relative speed of $11.7 \mathrm{~km} / \mathrm{s}$, destroying the two satellites. This event emphasized the need for increased accuracy in satellite orbit determination to help protect the investments of both commercial and government enterprises.

[3] Eventually, this research intends to increase the accuracy of satellite drag calculations, as well as improve understanding of the thermosphere wherein most satellites orbit. The immediate goal of this research is to demonstrate the effectiveness of using precision orbit measurements and ephemeris data to formulate corrections to existing

\footnotetext{
${ }^{1}$ Aerospace Engineering, University of Kansas, Lawrence, Kansas, USA.

Corresponding author: T. Lechtenberg, Graduate Research Assistant, Aerospace Engineering, University of Kansas, 1530 W 15th St., Lawrence, KS 66045, USA. (travis.lechtenberg@gmail.com)
}

(C2012. American Geophysical Union. All Rights Reserved. 1542-7390/13/2012SW000848 atmospheric density models. These corrections can be used to generate better atmospheric drag calculations, which will improve the accuracy of orbit determination and prediction, as well as increasing understanding of density variations in the upper thermosphere and exosphere. This paper examines density estimation for short-term density variations caused by coronal mass ejections, density increases near the geomagnetic poles of the earth, and midnight density maxima. Additionally, the errors incurred by altering the atmospheric neutral density values while propagating a satellite forward orbit are examined over periods of enhanced atmospheric variability.

[4] McLaughlin [2005] gives an introduction to the neutral atmosphere and the time-varying effects on thermospheric and exospheric density. These time-varying effects include solar rotation, the solar cycle, diurnal variations, magnetic storms and substorms, gravity waves, winds and tides, and long-term climate change. Vallado [2007] gives an introduction to the basic variations in density as well as the most commonly used density models in orbit determination. Hargreaves [1992] and Tribble [2003] give additional information on the space environment and neutral atmosphere. Sabol and Luu [2002] give a summary of the drivers of atmospheric density variations and discuss some of the difficulties caused by the lack of temporal resolution in geomagnetic and solar activity measurements utilized 


\section{LECHTENBERG ET AL.: THERMOSPHERIC DENSITY USING POES}

by the empirical density models. A discussion of the inaccuracies caused while modeling satellite drag is given by Marcos et al. [2003].

[5] The research presented in this paper is intended to examine the temporal resolution of POE-derived densities for later use in the orbit determination and density estimation of satellites that possess Global Positioning System (GPS) receivers and/or satellite laser ranging (SLR) retroreflectors. This research estimates the atmospheric density at altitude by using the post-processed precision orbit data of CHAMP in an orbit determination scheme and compares these densities to accelerometer-derived densities. Several previous papers have investigated the use of GPS receiver or SLR observations for estimating nonconservative accelerations. One approach is given by Doornbos et al. [2005] where a type of differential correction was examined using two-line element sets in a traditional dynamic calibration of the atmosphere (DCA) scheme along with a small number of satellites having precision orbit data to calibrate thermospheric neutral density models. Another approach, described as GPS accelerometry by van den IJssel and Visser [2005, 2007] and van den IJssel et al. [2005] utilizes GPS receiver data to estimate non-conservative forces as empirical accelerations acting on satellites. Using this method, the in-track and cross-track accelerations derived from the Challenging Minisatellite Payload (CHAMP) accelerometer may be reasonably determined with a temporal resolution of $20 \mathrm{~min}$ or less according to the authors.

[6] Previous work directly related to this research examined density estimation processes for the CHAMP and GRACE satellites. McLaughlin and Bieber [2008] examined the capability of POEs to characterize atmospheric density variations using the CHAMP satellite. McLaughlin et al. [2008] first compared results for POE-derived densities to densities derived from accelerometer measurements, and McLaughlin et al. [2011a] gave preliminary results of the calibration of ballistic coefficient and density half-lives that best corresponded to accelerometer derived density values. Hiatt et al. [2009] examined variations on the calibration results for periods of high-solar activity. Hiatt [2009] and Lechtenberg [2010] examined optimal half-life combinations for various combinations of solar and geomagnetic activity, as well as the observability of density variations. The research used precision orbit ephemeris (POE) data as observations in an optimal orbit determination scheme that estimated density and ballistic coefficient simultaneously. The density estimation was found to correlate quite well with densities found from the accelerometers onboard CHAMP and GRACE, but the temporal resolution of the density estimates was significantly worse than densities obtained from the accelerometers. Atmospheric density variations have also been examined in McLaughlin et al. [2011b], who looked at the variability of drag coefficients and hence, atmospheric density values over the course of 5 years for a select group of spherical satellites. This research will aim to better define the POE temporal density resolutions by examining CHAMP satellite density errors as compared to accelerometer derived densities. The POE-derived density estimates in previous works did not account for attitude or spacecraft geometry modeling, but relied strictly on the ballistic coefficient (BC) estimation. The resulting orbit position errors compared to the POE data were $3-8 \mathrm{~cm}$ for the processed data, which is within the accuracy of the POE data used as observations [Konig et al., 2002, 2005, 2006; Michalak et al., 2003].

[7] The CHAMP densities derived from POE data are compared to densities found from the accelerometers onboard the satellite. Accelerometers aboard satellites measure non-conservative accelerations, which can be utilized to estimate density using the drag equation. The accelerometer data allow for the separation of gravitational forces from non-conservative forces which include Earth radiation pressure, solar radiation pressure, and drag. Use of accurate radiation pressure models allows the drag acceleration and the resulting estimated density to be accurately calculated with relatively precise temporal resolution. Accelerometer data are extremely precise but available for only a few satellites, most notably CHAMP and GRACE. Konig and Neumayer (2003) and Bruinsma and Biancale [2003a] published some early results estimating atmospheric densities using CHAMP accelerometer data with additional, more precise atmospheric density values being later derived using CHAMP accelerometer data by Bruinsma and Biancale [2003b], Bruinsma et al. [2004], and Nerem et al. [2003]. Density variations often generate density waves that originate at high latitudes and then progress to lower latitudes. Typically, these waves dissipate at midrange latitudes, however, the waves take longer to dissipate if geomagnetic activity is high, and solar flux is low. Occasionally, coronal mass ejections (CME) impinge the atmosphere during these conditions, creating traveling atmospheric disturbances (TAD) that may be seen in satellite accelerometer observations [Bruinsma and Forbes, 2008, 2009]. Several papers involving joint work between researchers at the University of Colorado and the Centre National d'Études Spatiales (CNES) utilized CHAMP and GRACE accelerometer data to examine density variations created during solar and geomagnetic events [Sutton et al., 2005, 2006, 2007; Forbes et al., 2005; Bruinsma and Forbes, 2007; Bruinsma et al., 2006].

[8] Densities derived from POE are also compared to those from the High-Accuracy Satellite Drag Model (HASDM), which employs the dynamic calibration of the atmosphere (DCA) technique and applies it to a series of 75 inactive payloads, debris and satellites, including CHAMP [Storz et al., 2005]. HASDM is an initiative started by the Air Force Space Battlelab in January 2001 to improve Air Force Space Command's ability to meet Space Surveillance Capstone Requirements for satellite trajectory prediction accuracy. HASDM estimates and predicts, in $3 \mathrm{~h}$ updates, a constantly varying global density field. The model applies to satellites between 200 and $600 \mathrm{~km}$ in altitude and HASDM is capable of making predictions up to 3 days in advance. DCA utilizes the observed motions of a large number of satellites to estimate large-scale 


\section{LECHTENBERG ET AL.: THERMOSPHERIC DENSITY USING POES}

density corrections to a given atmospheric density model. Cefola et al. [2003], Yurasov et al. [2004, 2008], and Wilkins et al. [2007a, 2007b] are examples of the DCA approach of making large-scale corrections to existing atmospheric density models. DCA has shown remarkable ability to improve density estimation as compared to empirical models due to the model's use of a large number of calibration satellites to generate solutions. DCA generally utilizes two line element sets (TLEs) because tracking data is not readily available for a large number of objects. Two line element sets, or TLEs, are files containing sets of Keplerian orbital elements that define a satellite's orbit and position. Orbital elements are determined for many thousands of space objects by NORAD and are freely distributed on the Internet. HASDM uses primarily radar tracking data and high precision data that are incorporated into DCA solutions on a limited basis.

[9] Forbes et al. [2005] observed that during the period of 15-24 April 2002 several coronal mass ejections (CMEs) emanated from the Sun, coming into contact with the atmosphere and generating geomagnetic storms. These CMEs impinged the atmosphere and channeled energy into the upper atmosphere near the poles causing large localized density increases. These density increases propagated towards the equator as traveling atmospheric disturbances (TADs) in a wave-like fashion, with constructive interference occurring near the equator where the two waves propagating from opposing poles interacted. The magnitudes of these TADs were pronounced enough that they propagated past the opposing pole, and again toward their poles of origin; though, this effect was much less discernible than the initial waves.

[10] The density increases likely existed on both the lit and unlit sides of the Earth; however, they are much more apparent on the unlit side of the Earth where they are more easily separated from global density values. On the lit side of the Earth, atmospheric heating due to the Sun causes observation of these TADS to become more difficult.

[11] Using the STAR instrument aboard CHAMP, localized increases in thermospheric density were observed around the geomagnetic poles by Schlegel et al. [2005]. These localized densities demonstrated increases of up to $50 \%$ from ambient densities. The densities around the geomagnetic poles showed this increase at around $75^{\circ}$ geomagnetic latitude, with a basin localized around the actual geomagnetic pole. The first principle physics behind these increases is posited by Carlson et al. [2012] as requiring realistic transient shear velocities and $\mathrm{Ne}(\mathrm{h})$ profiles to properly model these density perturbations.

[12] The midnight density maximum (MDM) is a localized increase in density that occurs near the equator around midnight local time and is a byproduct of the midnight temperature maximum (MTM) caused by the convergence of thermospheric winds at low latitudes, which was first detected with ground based radar by Behnke and Harper [1973], Harper [1973], and Burnside et al. [1981]. The MDM has more recently been examined via satellite drag measurements by Arduini and Laneve [1997] using the San Marco 3 (SM3) and San Marco 5 (SM5) satellites using the two drag detectors each satellite possessed to determine local density variations along the low inclination satellite orbits. Faivre et al. [2006] examined climatology of the MTM, and hence, the MDM, using the Arequipa Fabry-Perot interferometer over the time period of 1997-2002, whenever local weather permitted. Akmaev et al. [2010] showed good agreement between the Whole Atmospheric Model (WAM) and in situ density observations, thus illustrating the precision with which the WAM operates. Observability of the MDM in CHAMP accelerometer-derived densities is alluded to in Liu et al. [2005] as part of a larger study of the global density distribution at $400 \mathrm{~km}$ in altitude. The accelerometers aboard the CHAMP spacecraft also displayed evidence of midnight density maxima, which occurred during the nocturnal passes of the satellite while passing over the equatorial plane. This density occurs over a period of roughly a few minutes during the satellite orbit, similar to the geomagnetic cusp phenomena.

[13] This paper has two goals: one, to examine density estimation for CHAMP on extremely short time scales, by examining events that show localized increases in density and using the temporal spans of these events to estimate the temporal resolution of the density corrections; and two, to show the effects of high frequency density variations on orbit propagation. Orbit propagation is the process by which the state of satellite is propagated forward or backward in time along its orbit using known force models. Previously, Anderson et al. [2009] looked at the effects on a propagated orbit due to the addition of cyclically varying parameters that effected drag, predominantly density, but also rapid impulse velocity changes. They showed that longer scale variations had a greater effect on orbit propagation than shorter scale variation. Schaeperkoetter and McLaughlin [2010] examined the propagated errors resulting from using a constant atmospheric density value or a sinusoidally varying density value, as well as the orbit accuracy of differing atmospheric density values for the CHAMP satellite at several different levels of both solar and geomagnetic activity. In the present paper, the orbits using the different densities are compared for $24 \mathrm{~h}$ propagation using both root mean square and maximum difference. This method does not account for how the difference in propagated position would give different model results. This is justified by assuming that density differences would be small.

\section{Methodology}

[14] The density estimation results presented in this paper were derived from precision orbit ephemerides (POE) for the CHAMP and GRACE satellites in an optimal determination process. The orbit determination process yielded density values along the path of the satellite, as well as ballistic coefficient values for the satellite during that time. POE data are currently available for both the CHAMP and GRACE satellites in the form of Postprocessed Science Orbits (PSO) or Rapid Science Orbits (RSO) from Helmholtz Centre Potsdam on their website 


\section{LECHTENBERG ET AL.: THERMOSPHERIC DENSITY USING POES}

at http://isdc.gfz-potsdam.de. Konig et al. [2002, 2005, 2006] and Michalak et al. [2003] discuss processing and accuracy of the RSOs. For the purposes of this research, RSO data are used due to its availability over the entire lifespan of the satellite.

[15] In this research, POE data were used as measurements in the optimal orbit determination scheme. These POEs provided measurements for use as input for a Kalman filter/smoother. A $90 \times 90$ GRACE Gravity Model 2 (GGM02C), solar radiation pressure, Earth infrared and albedo radiation pressure, luni-solar point masses, general relativity, and solid Earth and ocean tides are additional force models included in the orbit determination process.

[16] Wright [2003] and Wright and Woodburn [2004] outline the techniques for estimating density that are available in the Orbit Determination Tool Kit (ODTK) software package. The technique developed by Wright allows local atmospheric density to be estimated in real time in conjunction with the orbit determination process. Wright's technique provides significant improvement over the standard technique of estimating the ballistic coefficient (BC) or drag coefficient because BC estimates tend to absorb errors generated by the models for atmospheric density and BC. Additionally, BC estimates often include geopotential model errors. Wright demonstrated the feasibility of simultaneously observing both the atmospheric density and ballistic coefficient in a filtering scheme.

[17] Two corrections are made to the atmospheric density model that is used in ODTK; the first is a global correction to density based upon the daily $\mathrm{F}_{10.7}$ value, the daily $A_{p}$ value, and the height of perigee of the satellite orbit. The second correction accounts for sequential observations of the satellite which provide more up-to-date information of current atmospheric conditions. These sequential measurements take into account user-provided density and ballistic coefficient exponential Gauss-Markov process half-lives. The best set of density and ballistic coefficient correlation half-lives was found as $180 \mathrm{~min}$ for the density correlation half-life, and $1.8 \mathrm{~min}$ for the ballistic coefficient half-life with CIRA 1972 as a baseline density model by comparing POE-derived densities to accelerometer-derived densities for the CHAMP and GRACE satellites in Lechtenberg [2010], Fattig et al. [2010], and McLaughlin et al. [2010]. This is for a wide range of data points, and these density model parameters are used in the subsequent examinations of both the geomagnetic cusp data and midnight density maxima, unless otherwise noted. For the TAD data, the best combination of density and ballistic coefficient half-lives is found for the day in question using cross-correlation between the POE and accelerometer-derived densities as the judgment criteria. While the ballistic coefficient is estimated as part of the filter/smoother process, the ballistic coefficient was initialized using yearly averages of $0.00444 \mathrm{~m}^{2} / \mathrm{kg}$ for $2002-2003$ and $0.00436 \mathrm{~m}^{2} / \mathrm{kg}$ for 2004-2005 as determined by Bowman et al. [2008]. Values for the CHAMP satellite's nominal ballistic coefficient that were not included in these ranges were extrapolated to years both preceding and following these ranges by taking into account the changing mass of the satellite [Hiatt et al., 2009].

[18] The densities derived using POE data were compared to those derived from the CHAMP and GRACE accelerometers by Sean Bruinsma of CNES. The accelerometer derived densities are averaged over $10 \mathrm{~s}$ intervals [Bruinsma and Biancale, 2003a, 2003b; Bruinsma et al., 2004]. Previous analysis with CHAMP data that included a more representative analysis of days concluded that the optimal combinations of half lives and density models changed little as solar activity changed [Hiatt et al., 2009; Hiatt, 2009].

[19] The traveling atmospheric disturbances (TADs) that occur on the unlit portion of the Earth are observed by removing the portion of the satellite orbits that occur on the lit side of the Earth. This is determined using the local time stamps provided in the accelerometer density file in conjunction with the UTC time stamps that are supplied as part of the POE density determination scheme as well as the accelerometer-derived density file. The accelerometer-derived densities, POE-derived densities, and the densities derived by HASDM are compared to determine the observability of the TADs in the POE-derived densities and HASDM densities.

[20] Geomagnetic cusp features are localized around the geomagnetic poles, and thus in this examination, the latitudes and longitudes of the CHAMP satellite are converted into geomagnetic latitude and longitude. This was done by applying a three-dimensional polar coordinate transformation based upon the location of the geomagnetic poles for the year in question. The locations for the geomagnetic poles were obtained from values published by the Geological Survey of Canada [2008]. A series of geomagnetic polar passes are subsequently examined using this data, and the observability of this phenomenon using both POEderived density data and HASDM densities is assessed.

[21] The MDMs are found by examining both the POE and accelerometer-derived densities for dates during which both the CHAMP and GRACE satellites were coplanar and plotting them with an additional emphasis on the latitudinal effects on atmospheric density.

[22] In order to examine the effects of density model variation on satellite orbits, The POE, HASDM, and Jacchia 71 densities are normalized to the same mean as the accelerometer densities. The primary concern in this research is the effect of temporal variations on the orbit propagation and not on the bias between the density data sets or models. The normalization is performed by dividing the POE-derived, HASDM, or Jacchia 71 densities by their mean and multiplying by the mean of the accelerometer-derived densities. The means used are those for each particular $24 \mathrm{~h}$ period of propagation. This method of normalization was used assuming the bias between the data sets is caused by errors in the inverse ballistic coefficients used in solving for densities. During this examination, the values for the orbit propagation using the accelerometer derived densities are used as truth for comparison purposes. 


\section{LECHTENBERG ET AL.: THERMOSPHERIC DENSITY USING POES}

\section{Results}

[23] Five sets of results are presented. The first and second are density values for the TAD occurrences on 19 April 2002 and 23 May 2002. These are presented for the POE-derived densities, HASDM-derived CHAMP densities, and accelerometer-derived densities. The third set of results is for density values around selected observations of geomagnetic cusps. The fourth set represents the midnight density maxima and their observability in the POE densities, the empirical model, and in HASDM. The fifth and final set of results illustrates the effects of different density values on the orbit propagation values and the errors incurred by doing this.

\subsection{Traveling Atmospheric Disturbance Results}

[24] This section examines the observability of traveling atmospheric disturbances (TAD) during the time periods of 19 April 2002 and 23 May 2002. The temporal span of the disturbances, as measured by the satellite, was between 6 and $10 \mathrm{~min}$.

3.1.1. Density Values for Nocturnal Passes on 19 April 2002

[25] In Figure 1, the progression of the TAD as observed by the accelerometers onboard CHAMP on 19 April 2002 can be seen. In the top frame, the TAD has yet to initialize, and the atmospheric densities remain relatively constant. In the next frame, the TAD is propagating toward the equator and is observable as the two localized density increases at approximately $11.24 \mathrm{~h}$ and $11.46 \mathrm{~h}$. In the frame following this, the TADs from the opposing poles are constructively interfering near the equator, or at 12.9 $\mathrm{h}$. In the final frame, the TADs have passed through
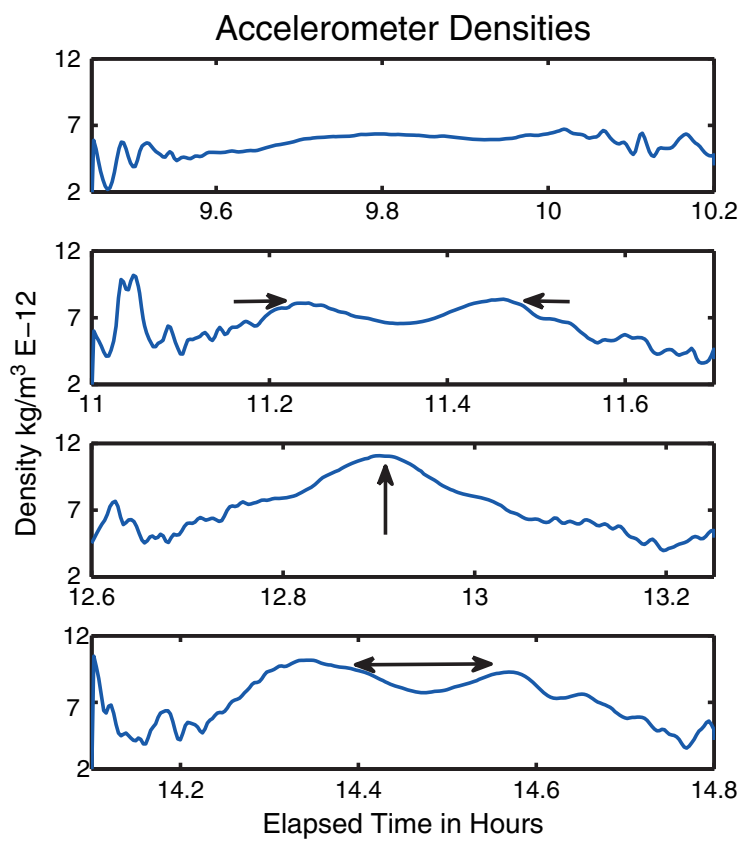

Figure 1. Nocturnal CHAMP satellite densities on 19 April 2002, Orbits 7-10. each other and continue on toward the opposing poles. The arrows in the frames indicate the TADs and the directions in which they are traveling. During the constructive interference phase, the arrow points directly upward, indicating the larger than normal response to the disturbance.

[26] Four density values were represented for each of the period of constructive interference in Figures 2. The first are the accelerometer densities, which are gauged as truth; the second are the densities predicted by HASDM; the third are densities obtained from the Jacchia 1970 empirical atmospheric model; and the final one was the best configuration of density and ballistic coefficient correlation half-lives determined in previous research, and discussed earlier. By examining these values, the degree to which the density models and modified density models characterize the TADs is found. There is a very minor possible response that may be observable as the two density waves approach each other in Figure 2, although as a whole, the density model's characterization of the TADs was very poor to non-existent.

\subsubsection{Density Values for Nocturnal Passes on} 23 May 2002

[27] In Figure 3, the progression of the TAD as observed by the accelerometers onboard CHAMP on 23 May 2002 can be seen. In the top frame, the TADs are seen at about $780 \mathrm{~min}$ and $805 \mathrm{~min}$, respectively, moving equator-ward. In the middle frame, the TADs are seen constructively interfering at about $880 \mathrm{~min}$ into the day. In the final frame, the TADs are moving past each other at this point and are visible at $975 \mathrm{~min}$ and $990 \mathrm{~min}$, respectively. The arrows in the frames have the same meanings as in Figure 1, indicating the location and direction of the TADs.

[28] Four density values were represented in Figure 4 during the nocturnal passes on 23 May 2002 for the pass containing the constructive interference seen by the accelerometers aboard CHAMP. The first are the accelerometer densities, which are gauged as truth; the second are the densities predicted by HASDM; the third are densities

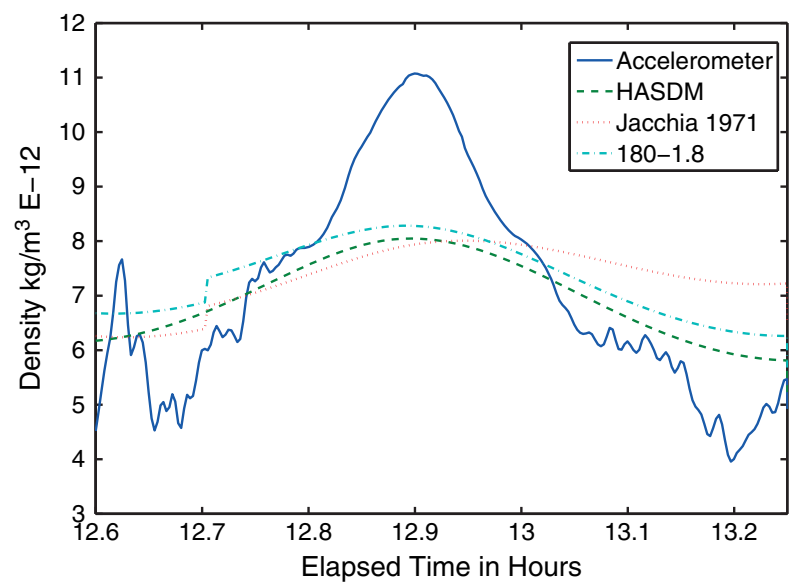

Figure 2. Comparison of Nocturnal CHAMP satellite densities on 19 April 2002, Orbit 8. 

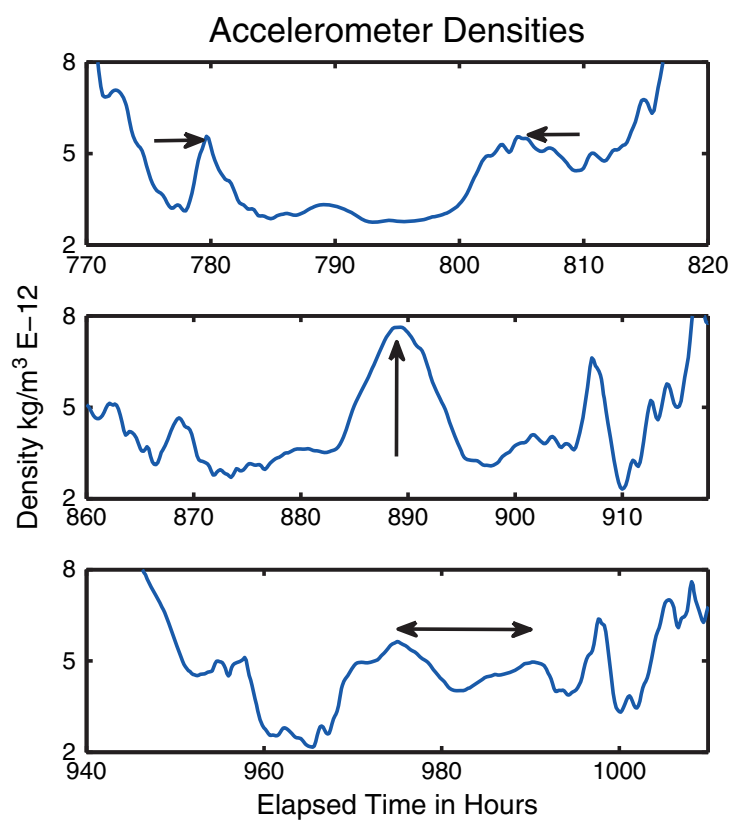

Figure 3. Nocturnal CHAMP satellite densities on 23 May 2002, Orbits 9-11.

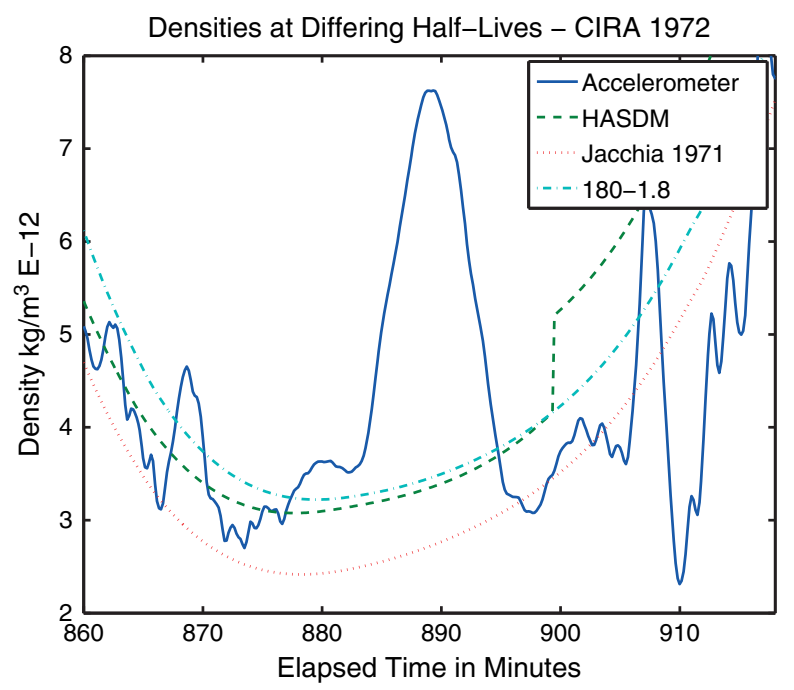

Figure 4. Comparison of nocturnal CHAMP satellite densities on 23 May 2002, Orbit 10.

obtained from the empirical Jacchia 1970 atmospheric model; and the final set of densities is again the optimal configuration determined for overall data. Examination of these values will indicate if the lack of correlation in the previous subsection was merely an outlier in terms of the prediction of atmospheric density for TADs.

[29] As with the TADs seen on 19 April, none of the density models displayed in Figure 4 showed any capability to model the traveling atmospheric disturbances. While the density model results for 19 April showed what might be construed as a minor response to the TADs, the density model response for 23 May showed no indication of responding to the TADs. The model densities for 23 May decreased smoothly to a minimum value early in the nocturnal part of the orbit and then rose again in response to diurnal heating.

\subsection{Geomagnetic Cusp Results}

[30] In this section, the observability of localized increases to atmospheric density near the north geomagnetic pole is examined for selected geomagnetic polar passes that show significant and noticeable increases in density. This will be examined by using graphical representations of the CHAMP satellite's geomagnetic latitude, as well as a graphical representation of density values determined by the onboard accelerometer, HASDM, and the POE-derived densities described earlier in this work. Only POE data corresponding to the best orbit determination configurations for all levels of solar and geomagnetic activity are used.

[31] Densities derived from HASDM and POE are much closer to values for atmospheric density derived from accelerometer measurements than the empirical Jacchia 1971 model, although none of the density estimates show any indication of modeling this geomagnetic cusp phenomenon in Figure 5.

[32] Again, HASDM and POE-derived densities are much closer to values for atmospheric density derived from accelerometer measurements than the empirical Jacchia 1971 model, and none of the density estimates show any indication of modeling the geomagnetic cusp phenomena in Figure 6.

[33] All density models show a very minor peak near the maximum latitude the satellite reaches. However, this does not correspond with either of the density increases on either side of this peak in Figure 7.

[34] All density models again show a minor peak following the first pass over the geomagnetic cusp, although the depression between the geomagnetic cusps is not

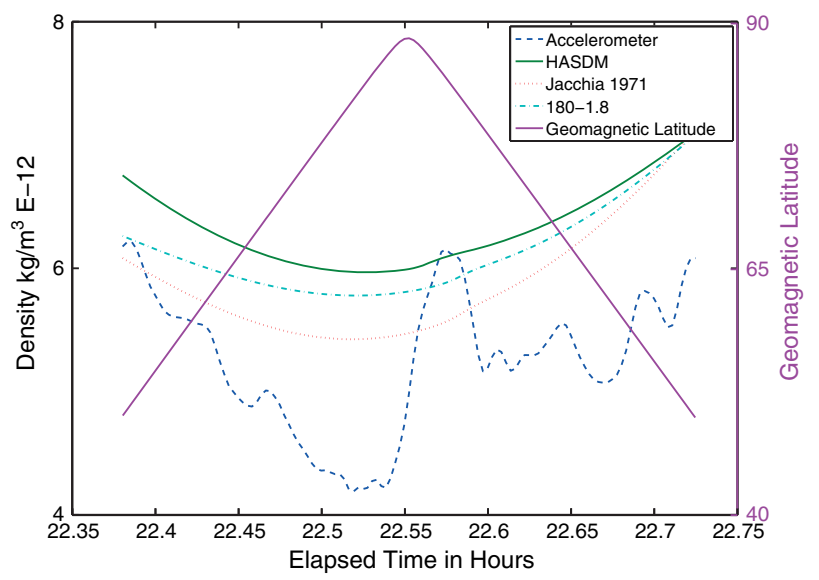

Figure 5. CHAMP geomagnetic pole pass at approximately 22:30 UTC 19 April 2002; an atmospheric density peak is observable at 22:33 UTC. 


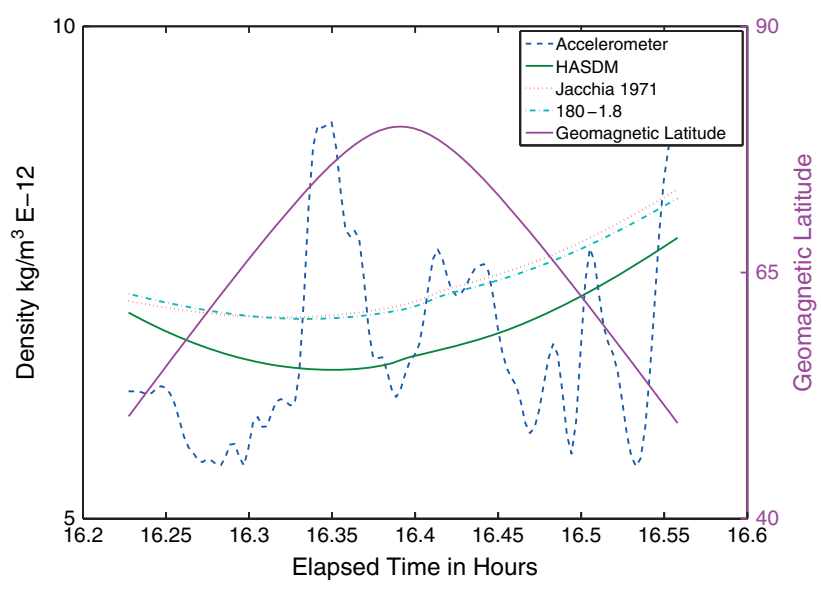

Figure 6. CHAMP geomagnetic pole pass at approximately 16:24 UTC 19 April 2002; atmospheric density peaks are observable in accelerometer data at 16:21 and 16:26 UTC.

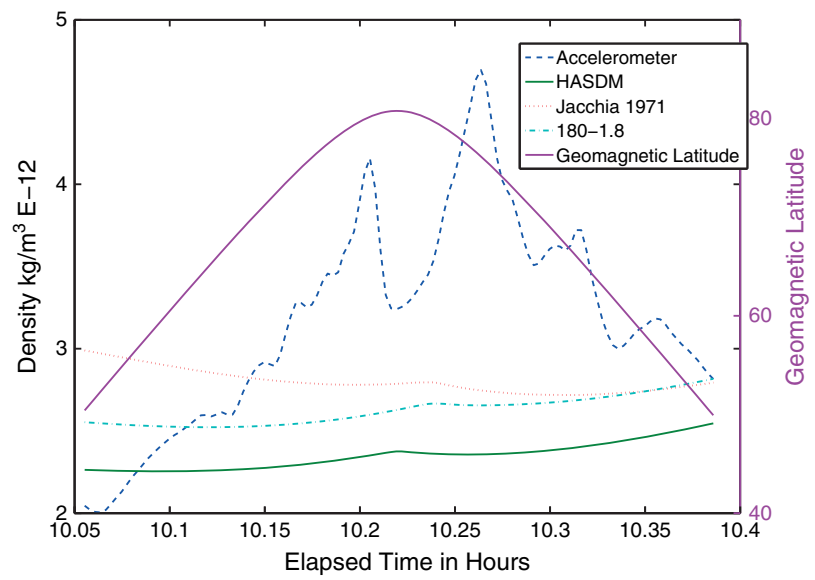

Figure 7. CHAMP geomagnetic pole pass at approximately 10:14 UTC 21 March 2003; atmospheric density peaks are observable in accelerometer data at 10:12 and 10:16 UTC.

characterized in any of the density models in Figure 8. The density models show a significant increase in density following the pass over the geomagnetic pole as the CHAMP satellite moves to the lit side of the Earth.

\subsection{Midnight Density Maxima}

[35] In this section, the local increases in density that occur near or at the equator around midnight local time are examined for their observability in POE derived densities, as well as the empirical density models, and HASDM. Only POE data corresponding to the best orbit determination configuration for all levels of solar and geomagnetic activity are used.

[36] In Figure 9 localized increases in density can be seen in the troughs near the equator, and minor response can be seen in the POE densities, empirical model, and HASDM.

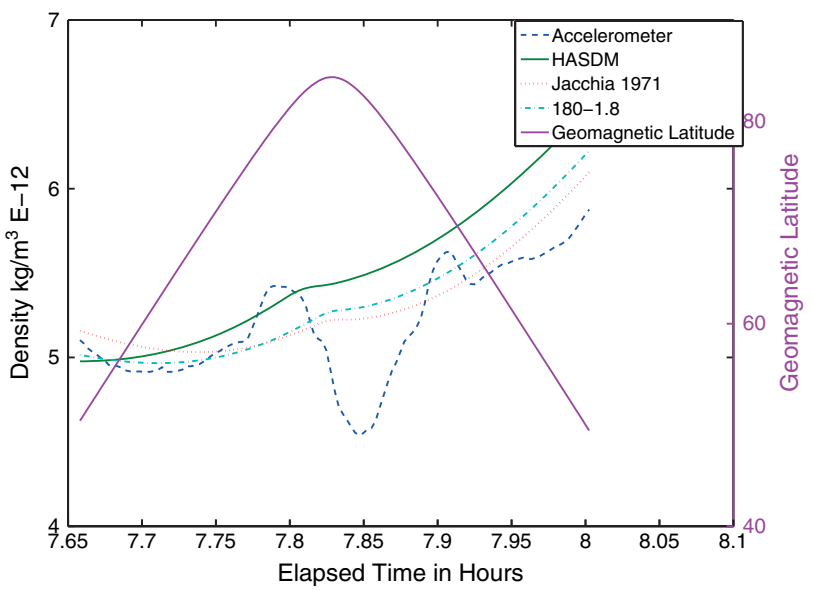

Figure 8. CHAMP geomagnetic pole pass at approximately 7:50 UTC 19 February 2002; atmospheric density peaks are observable in accelerometer data at 10:12 and 10:16 UTC.

The non-accelerometer-derived densities do not show the same degree of variability as the accelerometer-derived densities, although HASDM has an apparent secondary maximum at a time of $1040 \mathrm{~min}$ that seems to correspond well to the MDM seen in the accelerometer densities.

\subsection{Orbit Propagation using Differing Density Values}

[37] The errors in orbit propagation caused by density errors are examined in this section for normal days, days in which TADs were observed, days during which geomagnetic cusp features were observed for the CHAMP satellite, days in which MDMs have been observed, and only for a normal day for the GRACE satellite. These orbits are propagated using a precise initial vector of position

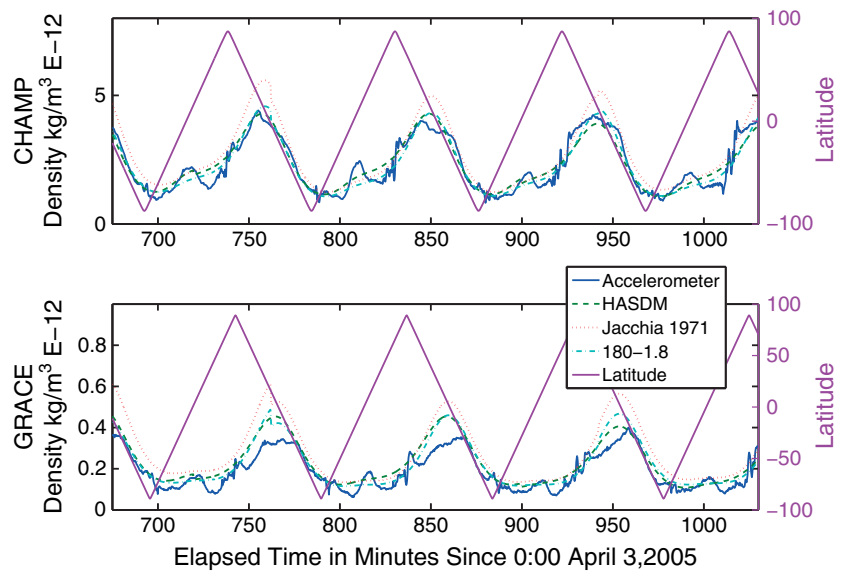

Figure 9. CHAMP and GRACE neutral densities during the coplanar period of 5 April 2005; localized density maxima can be seen in the troughs between peaks near a latitude of about 0 , these maxima can be seen near times of 1040, 1135, 1225, and 1320. 


\section{LECHTENBERG ET AL:: THERMOSPHERIC DENSITY USING POES}

and velocity, and then atmospheric density values from the various sources are used in the orbit propagation scheme.

[38] Figure 10 shows the densities and orbit propagation results for a normal day for CHAMP. The densities in the figure are the actual model or derived densities that have not been normalized. The reason for plotting the densities before normalization is so the different density sources can be more easily observed in the figure. The POEderived densities clearly provide better orbit propagation accuracy than HASDM or Jacchia 71. The worst case error for the propagation with POE derived densities is only $13 \mathrm{~m}$, which would not be significant for most applications.

[39] Figure 11 includes densities and orbit propagation errors for a day that includes a TAD; the figure plots the

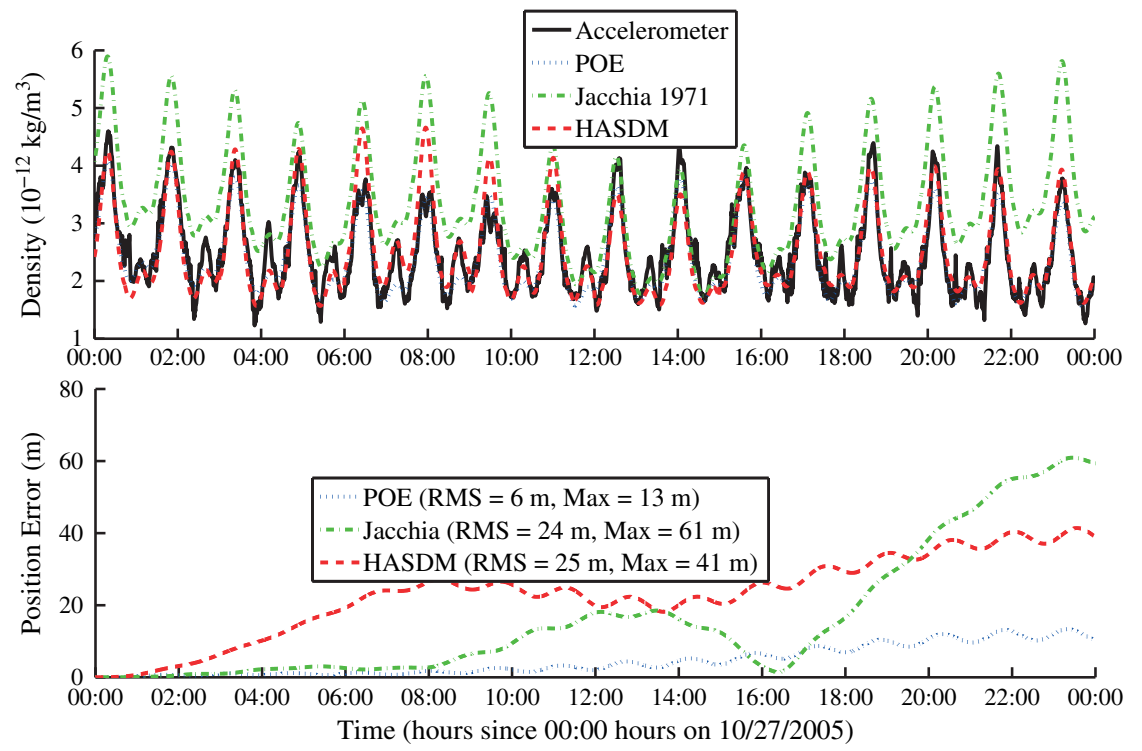

Figure 10. Density and orbit propagation errors along the CHAMP orbit on a normal day; the position errors slowly accumulate, with the Jacchia 1971 model eventually having the largest accumulated errors.
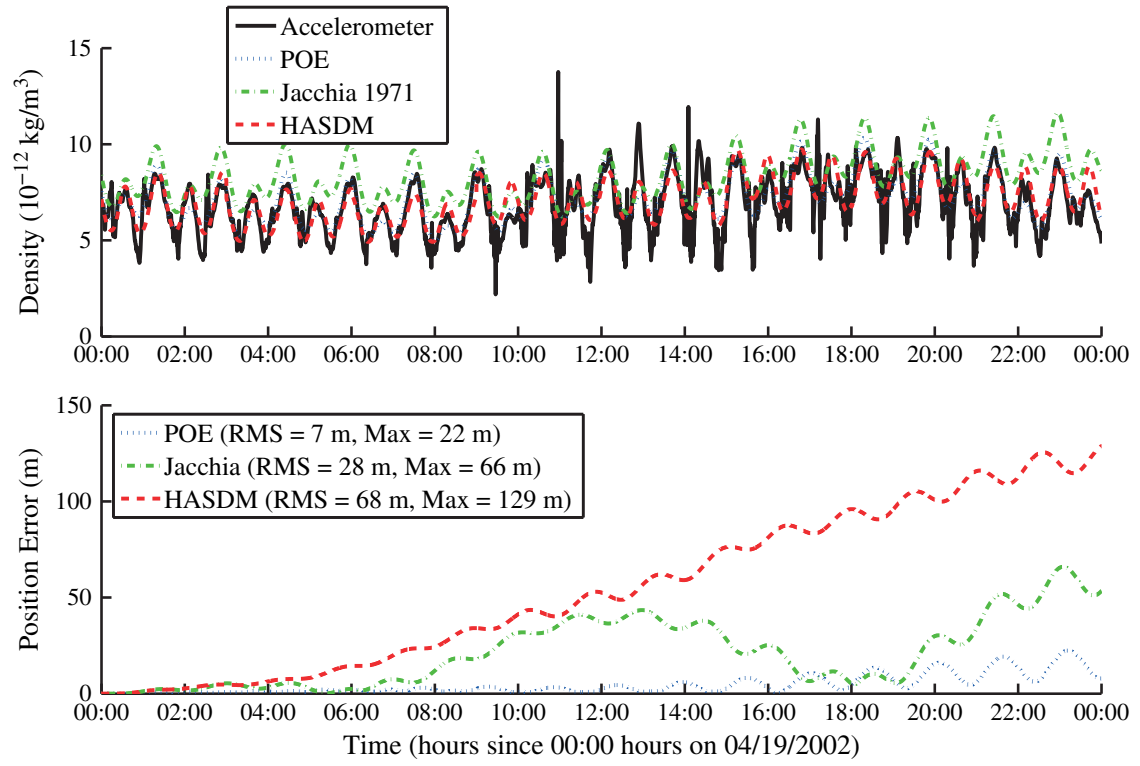

Figure 11. Density and orbit propagation errors along the CHAMP orbit on a day with a traveling atmospheric disturbance (TAD); the errors slowly accumulate over the course of the satellite arc, with the POE-derived densities eventually having the least accumulated errors, and the HASDM densities having the most. 


\section{LECHTENBERG ET AL.: THERMOSPHERIC DENSITY USING POES}

direction of the drift caused by errors in the Jacchia 71 model changes at about this time. However, the errors in orbit propagation using the POE-derived densities remain quite small. Clearly, the high frequency signals observed by the accelerometer, but not in the POE-derived densities do not have a major effect on orbit propagation.

[40] The STAR accelerometer on CHAMP observed density enhancements that occurred around the polar cusp. Figure 12 shows densities and orbit errors for 21 March 2002, which was 1 day on which geomagnetic cusp enhancements were observed. A small spike in the accelerometer density can be seen at this time, but no discernible effects are observed on any of the orbit propagations. The maximum error for the orbit propagation with the POE derived densities only reaches $8 \mathrm{~m}$.

[41] Midnight density maxima are observable in the CHAMP accelerometer-derived densities on 3 April 2005. Figure 13 displays the densities and propagated orbit errors for this day, where the effects of the midnight density maximum are unable to be observed due to the dominance of diurnal heating and atmospheric expansion. The POE-derived densities perform better than those found using HASDM, however, over the course of the day, the Jacchia 1971 densities eventually yield better results despite showing more pronounced errors between the hours of 5:00 and 12:00.

[42] Figure 14 shows the densities and orbit errors for a normal day for GRACE. The orbit errors caused by density errors are also considerably lower for GRACE than for CHAMP because of its higher altitude. The maximum orbit propagation errors here are all less than $10 \mathrm{~m}$. However, these are errors for densities that have been normalized. The Jacchia 71 model would clearly have higher errors without the normalization because of the bias between the Jacchia 71 densities and the other densities that appear in the upper plot of Figure 17.

\section{Conclusion}

[43] Precision orbit ephemeris data were used as observations in an optimal orbit determination scheme to estimate density. The density values found for CHAMP orbits during the examined times and occurrences were then compared to the accelerometer densities to determine accuracy.

[44] There is relatively little to suggest that either the POE-derived densities, the HASDM densities, or the empirical model densities are able to predict the appearance and characteristics of traveling atmospheric disturbances. The empirical model is not expected to display this behavior as it does not account for satellite measurements at all. Both the empirical model and HASDM are included for purposes of comparison and to demonstrate the effects of the underlying model. There are no localized increases in density that correspond to the increases seen in accelerometer data due to the traveling atmospheric disturbances. During the period of constructive interference, the peak amplitudes of both POE-derived densities and HASDM densities do seem to correspond with the peak associated with the constructive interference, although that peak appears to simply be a product of the density models as it appears in the other three nocturnal passes as well.

[45] The POE-derived densities have a difficult time modeling geomagnetic cusp features as indicated by the lack of corresponding density peaks. Both the empirical
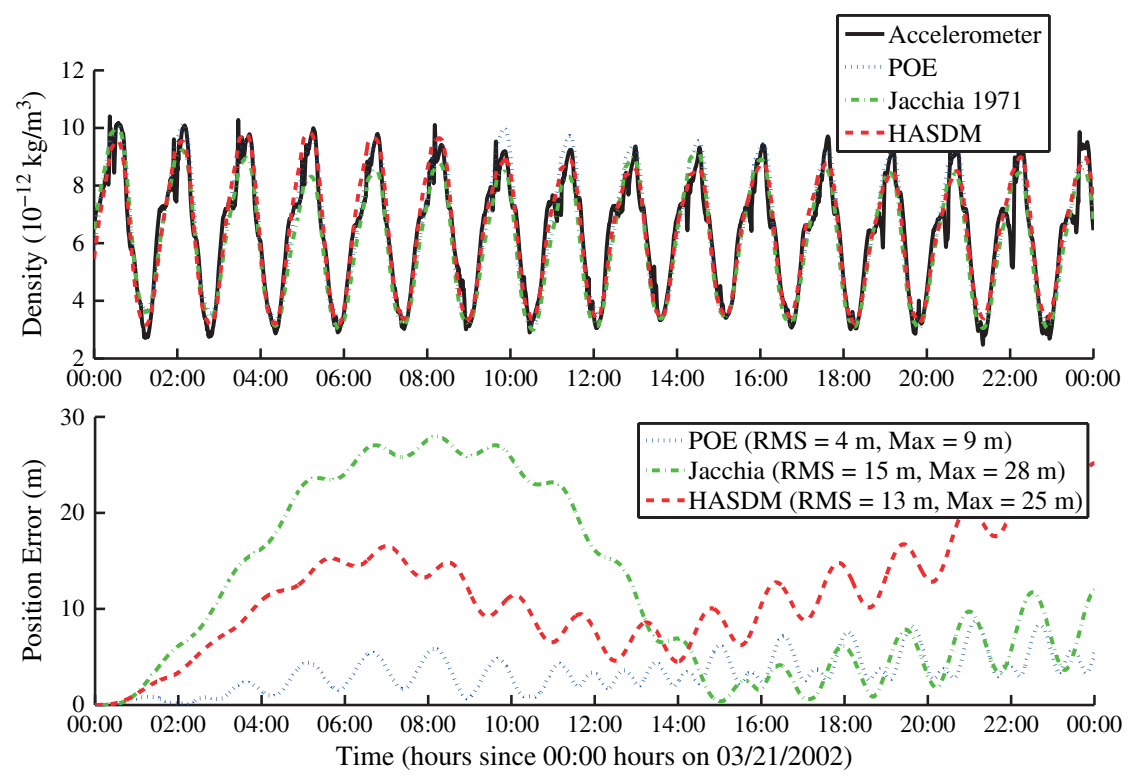

Figure 12. Density and orbit propagation errors along the CHAMP orbit on a day with a geomagnetic cusp enhancement; the errors slowly accumulate over the course of the satellite arc, with the POE derived densities eventually having the least accumulated errors, and the HASDM densities having the most. 

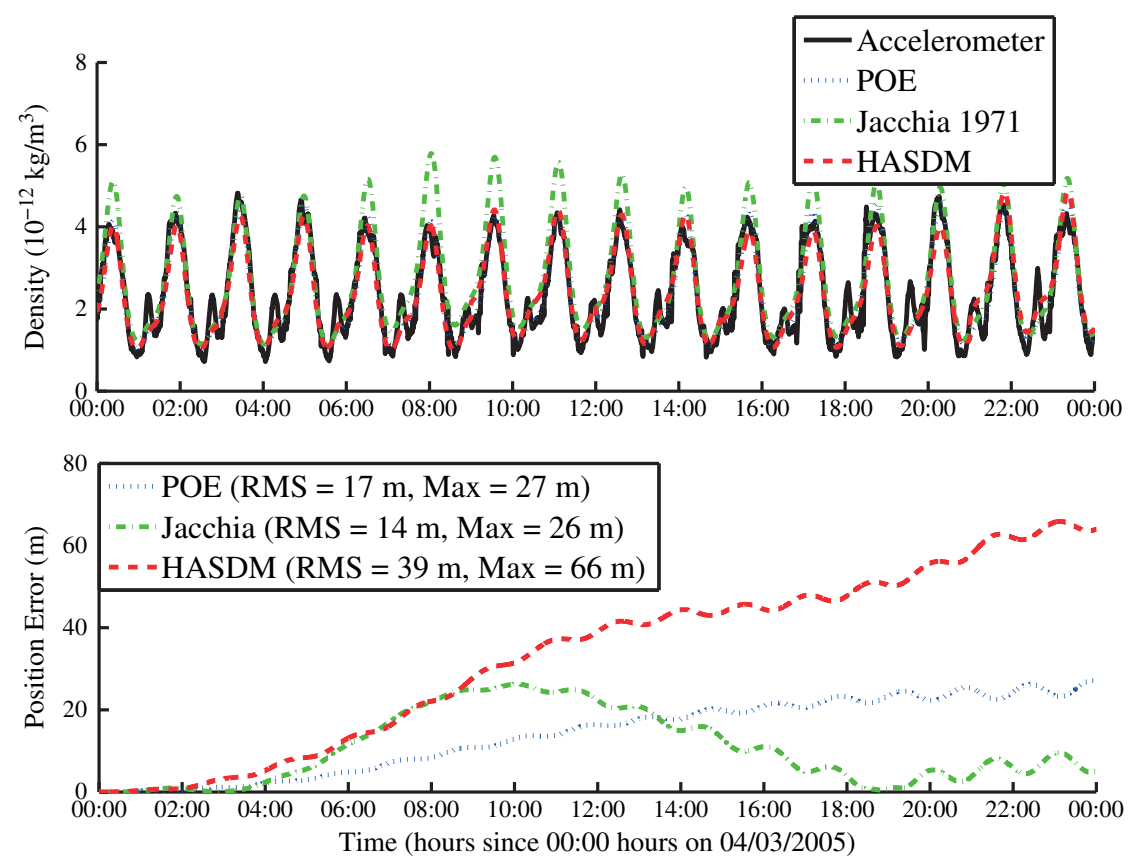

Figure 13. Density and orbit propagation errors along the CHAMP orbit on a day previously observed to illustrate the phenomenon of midnight density maxima; the errors build up with HASDM showing the poorest results, and the Jacchia 1971 showing the best.

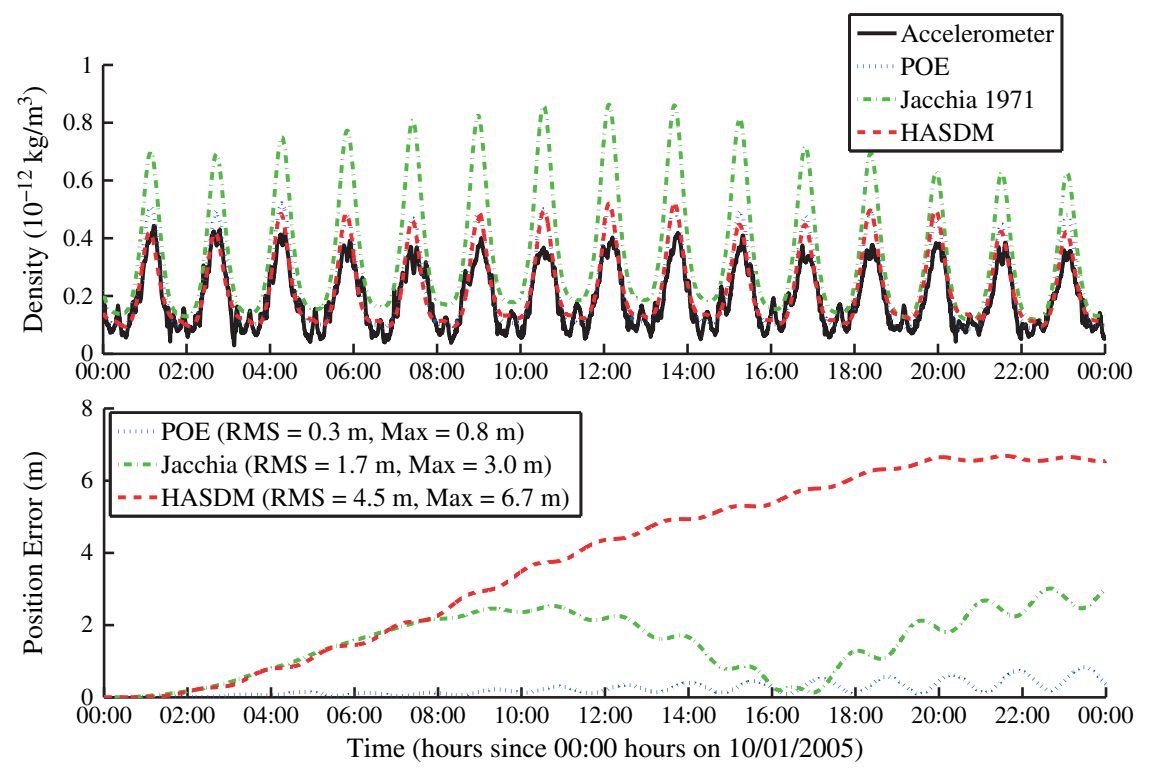

Figure 14. Density and orbit propagation errors along the GRACE orbit on a normal day; GRACE shows similar results to those of CHAMP, with HASDM having the largest accumulated errors, and the POE-derived densities having the least.

Jacchia 1971 and HASDM densities also do very little to model these very short term perturbations. At some points, the geomagnetic cusps are observable in the accelerometer data on either side of the geomagnetic pole, and at one point the cusp is only seen on the later side of the geomagnetic pole. Currently, the temporal resolution of these density models is obviously not of sufficient quality to model these density perturbations.

[46] The minor responses to the midnight density maxima indicate that the models and POE-derived 


\section{LECHTENBERG ET AL.: THERMOSPHERIC DENSITY USING POES}

densities currently do not adequately model the MDMs. The minor response also indicates that there may be corrections that may be made to better model the MDMs. HASDM shows slightly higher response to the MDMs during one trough area in Figure 13, although the model is inconsistent in its modeling of the minor increases in density. Overall, the temporal resolution of the densities was insufficient to model the perturbations that arise at the midnight density maxima.

[47] The effect of density estimation on orbit accuracy was examined to show the impact of the temporal resolutions of the models. The POE-derived densities were superior for orbit propagation compared to Jacchia 71 and HASDM in all cases. The errors between the orbits propagated with POE-derived densities compared to orbits propagated with accelerometer-derived densities were around $1 \mathrm{~m}$ for GRACE and ranged from 4 to $22 \mathrm{~m}$ for CHAMP. These errors are not significant for most orbit applications. Therefore, the high frequency variations observed only by the accelerometer are not significant for most orbit analysis applications.

[48] Acknowledgments. Any opinions, findings, and conclusions or recommendations expressed in this material are those of the authors and do not necessarily reflect the views of the National Science Foundation. Funding for this work was provided the National Science Foundation award \#0832900 with some additional support provided by the Kansas Space Grant Consortium. David Vallado's help and expertise in working with data conversion scripts and with the Orbit Determination Tool Kit (ODTK) was invaluable. Aid with ODTK scripting was provided by Jens Ramrath at Analytical Graphics, Inc. (AGI). Accelerometer-derived densities were provided by Sean Bruinsma of the Centre National d'Études Spatiales (CNES) and density values for the High Accuracy Satellite Drag Model (HASDM) were provided by Bruce Bowman of the U.S. Air Force.

\section{Reference}

Anderson, R. L., G. H. Born, and J. R. Forbes (2009), "Sensitivity of orbit predictions to density variability," J. Spacecraft Rockets, 46, No. 6, November-December 2009, 1214-1230.

Akmaev, R. A., F. Wu, T. U. Fuller-Rowell, H. Wang, and M. D. Iredell (2010), "midnight density and temperature maxima, and thermospheric dynamics in whole atmosphere model simulations," J. Geophys. Res., 115, doi:10.1029/2010JA015651.

Arduini, C. and G. Laneve (1997), "Local time and altitude variation of equatorial thermosphere midnight density maximum (MDM): San Marco drag balance measurements," Geophys. Res. Lett., 24, No. 4, 377-380.

Behnke, R. A., and R. M. Harper (1973), "Vector measurements of $F$ region ion transport at Arecibo," J. Geophys. Res., 78, 8222-8234.

Bowman B. R., F. A. Marcos, K. Moe, M. M. Moe (2008), “Determination of drag coefficient values for CHAMP and GRACE satellites using orbit drag analysis," Adv. Astronaut. Sci., 129, AAS 07-259, Univelt, $147-166$

Bruinsma S. and R. Biancale (2003a), "Total Density Retrieval with STAR," First CHAMP mission results for gravity, magnetic and atmospheric studies, eds. C. Reigber, H. Luhr, P. Schwintzer, Springer, Berlin, 192-199.

Bruinsma S. and R. Biancale (2003b), "Total densities derived from accelerometer data," Journal of Spacecraft and Rockets, 40, No. 2, March-April 2003, 230-236.

Bruinsma S. L. and J. M. Forbes (2007), "Storm-time equatorial density enhancements observed by CHAMP and GRACE," Journal of Spacecraft and Rockets, 44, No. 6, 1154-1159.
Bruinsma S. L. and J. M. Forbes (2008), "Medium- to large-scale density variability as observed by CHAMP," Space Weather, 6, S08002, doi:10.1029/2008SW000411.

Bruinsma S. L. and J. M. Forbes (2009), "Properties of traveling atmospheric disturbances (TADs) inferred from CHAMP accelerometer observations," Adv. Space Res., 43, 369-376.

Bruinsma S., S. D. Tamagnan and R. Biancale (2004), "Atmospheric densities derived from CHAMP/STAR accelerometer observations," Planet. Space Sci., 52, 297-312.

Bruinsma S., J. M. Forbes, R. S. Nerem, and X. Zhang (2006), "Thermospheric density response to the 20-21 November 2003 solar and geomagnetic storm from CHAMP and GRACE accelerometer data," J. Geophys. Res., 111, No. AO6303, 1-14.

Burnside, R. G., F. A. Herrero, J. W. Meriwether, and J. C. G. Walker (1981), "Optical observations of thermospheric dynamics at Arecibo," J. Geophys. Res., 86, 5532-5540.

Carlson, H. C., T. Spain, A. Aruliah, A. Skjaeveland, and J. Moen (2012), "First-principles physics of cusp/polar cap thermospheric disturbances," Geophys. Res. Lett., 39, doi:10.1029/ 2012GL053034.

Cefola P. J., R. J. Proulx, A. I. Nazarenko, and V. S. Yurasov (2003), "Atmospheric density correction using two line element sets as the observation data," Adv. Astronaut. Sci., 116, AAS 03-626, Univelt, 1953-1978.

Doornbos E., H. Klinkrad, and P. Visser (2005), "Atmospheric density calibration using satellite drag observations," Adv. Space Res., Vol. 36, 515-521.

Faivre, M., J. W. Meriwether, C. G. Fesen, and M. A. Biondi (2006), "Climatology of the midnight temperature maximum phenomenon at Arequipa, Peru," J. Geophys. Res., Vol 111, doi:10.1029/ 2005JA011321.

Fattig, E., McLaughlin, C. A., and T. Lechtenberg (2010), “Comparison of density estimation for CHAMP and GRACE satellites," AIAA/ AAS Astrodynamics Specialist Conference, Toronto, ON, AIAA 2010-7976, 2-5 August 2010.

Forbes J. M., G. Lu, S. Bruinsma, S. Nerem, and X. Zhang (2005), "Thermospheric density variations due to the 15-24 April 2002 solar events from CHAMP/STAR accelerometer measurements," J. Geophys. Res., 110, 1-9.

Geological Survey of Canada (2008), “Geomagnetism: North magnetic pole," Jan. 2008, Last Accessed: 28 March 2010, http://gsc.nrcan.gc. ca/geomag/nmp/northpole_e.php.

Hargreaves J. K. (1992), The Solar-Terrestrial Environment, Cambridge University Press, Cambridge.

Harper, R. M. (1973), “Nighttime meridional neutral winds near $350 \mathrm{~km}$ at low to mid-latitudes," J. Atmos. Terr. Phys., 35, 2023-2034.

Hiatt A. (2009), "Deriving atmospheric density estimates using satellite precision orbit ephemerides," MS Thesis, Department of Aerospace Engineering, University of Kansas.

Hiatt A., C. A. McLaughlin, T. Lechtenberg (2009), “Deriving density estimates using CHAMP precision orbit data for periods of high solar activity," Adv. Astronaut. Sci., 134, AAS 09-104, Univelt, 23-42.

Konig R. and K. H. Neumayer (2003), "Thermospheric events in CHAMP precise orbit determination," First CHAMP Mission Results for Gravity, Magnetic and Atmospheric Studies, eds. C. Reigber, H. Luhr, P. Schwintzer, Springer, Berlin, 112-119.

Konig R., S. Zhu, C. Reigber, K. H. Neumayer, H. Meixner, R. Galas, G. Baustert (2002), "CHAMP rapid orbit determination for GPS atmospheric limb sounding," Adv. Space Res., 30, No. 2, 289-293.

Konig R., G. Michalak, K. H. Neumayer, S. Y. Zhu, H. Meixner, C. Reigber (2005), "Recent developments in CHAMP orbit determination at GFZ," Earth Observation with CHAMP Results from Three Years in Orbit, eds. C. Reigber, H. Luhr, P. Schwintzer, J. Wickert, Springer, Berlin, 65-70.

Konig R., G. Michalak, K. H. Neumayer, S. Zhu (2006), "Remarks on CHAMP orbit products," Observation of the Earth System from Space, eds. J. Flury, R. Rummel, C. Reigber, M. Rothacher, G. Boedecker, U. Schreiber, Springer, Berlin, 17-26.

Lechtenberg T. (2010), "Derivation and observability of upper atmospheric density variations utilizing precision orbit ephemerides," MS Thesis, Department of Aerospace Engineering, University of Kansas. 


\section{LECHTENBERG ET AL.: THERMOSPHERIC DENSITY USING POES}

Liu, H., H. Lühr, V. Henize, and W. Köhler (2005), “Global distribution of the thermospheric total mass density derived from CHAMP," J. Geophys. Res., 110, doi:10.1029/2004JA010741.

Marcos F. O., J. O. Wise, M. J. Kendra, and N. J. Grossbard (2003), "Satellite drag research: Past, present, and future," Adv. Astronaut. Sci., 116, AAS 03-620, Univelt, 1865-1878.

McLaughlin C. A. (2005), “Upper atmospheric phenomena and satellite drag," Adv. Astronaut. Sci., 123, AAS 05-315, Univelt, 989-996.

McLaughlin C. A. and B. S. Bieber (2008), "Neutral density determined from CHAMP precision orbits," Adv. Astronaut. Sci., 129, AAS 07-260, Univelt, 167-186.

McLaughlin C. A., A. Hiatt, and B. S. Bieber (2008), "Comparison of total density derived from CHAMP precision orbits and CHAMP accelerometer," Adv. Astronaut. Sci., 130, AAS 08-177, Univelt, 1193-1206.

McLaughlin, C. A., T. Lechtenberg, and E. Fattig (2010), “Estimating density using precision satellite orbits from multiple satellites," Kyle T. Alfriend Astrodynamics Symposium, 139 of Advances in the Astronautical Sciences, 2010, AAS 10-307, 87-102.

McLaughlin C. A., A. Hiatt, and T. Lechtenberg (2011a), "Precision orbit derived total density," Journal of Spacecraft and Rockets, 48, No. 1, January-February, 2011, 166-174.

McLaughlin, C. A., S. Mance, and T. Lechtenberg (2011b), “Drag coefficient estimation in orbit determination," J. Astronaut. Sci., 58, No. 3, July-September 2011, 513-530.

Michalak G., G. Baustert, R. Konig, C. Reigber (2003), "CHAMP rapid science orbit determination: Status and future prospects," First CHAMP Mission Results for Gravity, Magnetic and Atmospheric Studies, eds. C. Reigber, H. Luhr, P. Schwintzer, Springer, Berlin, 98-103.

Nerem R. S., J. M. Forbes, E. K. Sutton, and S. Bruinsma (2003), "Atmospheric density measurements derived from CHAMP/STAR accelerometer data," Adv. Astronaut. Sci., 116, AAS 03-621, Univelt, 1879-1898.

Sabol C. and K. K. Luu (2002), "Atmospheric density dynamics and the motion of satellites," AMOS Technical Conference, Wailea, HI, September.

Schaeperkoetter, A., C. McLaughlin (2010), "Effects of density variations in the upper atmosphere on satellite trajectories," Space Flight Mechanics 2010, 136 of Advances in the Astronautical Sciences, 2010, AAS 10-223, 1817-1830.

Schlegel K., H. Luhr, J. P. St. Maurice, G. Crowley, and C. Hackert (2005), "Thermospheric density structures over the polar regions observed with CHAMP," Ann. Geophys., 23, 1659-1672.
Storz M. F., B. R. Bowman, Major J. I. Branson, S. J. Casali, and W. K. Tobiska (2005), "High accuracy satellite drag model (HASDM)," Adv. Space Res., 36, Issue 12, 2497-2505.

Sutton E. K., R. S. Nerem, and J. M. Forbes (2005), “Global thermospheric neutral density and wind response to the severe 2003 geomagnetic storms from CHAMP accelerometer data," J. Geophys. Res., 110, doi:10.1029/2004JA010985.

Sutton E. K., J. M. Forbes, R. S. Nerem, and T. N. Woods (2006), “Neutral density response to the solar flares of October and November, 2003," Geophys. Res. Lett., 33, doi:10.1029/2006GL027737.

Sutton E. K., R. S. Nerem, and J. M. Forbes (2007), "Density and winds in the thermosphere deduced from accelerometer data," Journal of Spacecraft and Rockets, 44, No. 6, 1210-1219.

Tribble A. C. (2003), The Space Environment: Implications for Spacecraft Design, Princeton University Press, Princeton, New Jersey.

Vallado D. A. (2007), Fundamentals of Astrodynamics and Applications, Microcosm Press, El Segundo, CA, 3rd Edition, Chap. 8, App. B.

van den IJssel J. and P. Visser (2005), "Determination of non-gravitational accelerations from GPS satellite-to-satellite tracking of CHAMP," Adv. Space Res., 36, 418-423.

van den IJssel J. and P. Visser (2007), "Performance of GPS accelerometry: CHAMP and GRACE," Adv. Space Res., 39, 1597-1603.

van den IJssel J., P. Visser, and R. Haagmans (2005), "Determination of non-gravitational accelerations from orbit analysis," Earth Observation with CHAMP Results from Three Years in Orbit, eds. C. Reigber, H. Luhr, P. Schwintzer, J. Wickert, Springer, Berlin, 95-100.

Wilkins M. P., C. A. Sabol, P. J. Cefola, and K. T. Alfriend (2007a), "Improving dynamic calibration of the atmosphere," Adv. Astronaut. Sci., 127, AAS 07-185, Univelt, 1257-1272.

Wilkins M. P., C. A. Sabol, P. J. Cefola, and K. T. Alfriend (2007b), "Validation and application of corrections to the NRLMSISE-00 atmospheric density model," Adv. Astronaut. Sci., 127, AAS 07-189, Univelt, 1285-1304.

Wright J. R. (2003), "Real-time estimation of local atmospheric density," Adv. Astronaut. Sci., 114, AAS 03-164, Univelt, 927-950.

Wright J. R. and J. Woodburn (2004), "Simultaneous real-time estimation of atmospheric density and ballistic coefficient," Adv. Astronaut. Sci., 119, AAS 04-175, Univelt, 1155-1184.

Yurasov V. S., A. I. Nazarenko, P. J. Cefola, and K. T. Alfriend (2004), "Results and issues of atmospheric density correction," J. Astronaut. Sci., 52, No. 3, July-September 2004, 281-300.

Yurasov V. S., A. I. Nazarenko, K. T. Alfriend, and P. J. Cefola (2008), "Reentry Time prediction using atmospheric density corrections," J. Guid. Control Dyn., 31, No. 2, March-April 2008, 282-289. 